\title{
Enfermedad de Wilson: reporte de caso
}

\author{
Wilson's disease: a case report \\ Guartazaca Guerrero Erika Paola', León Domínguez Cristina \\ Elizabeth', Orellana Cobos Danilo Fernando?.
}

VOLUMEN 37 | N 3 | DICIEMBRE 2019

FECHA DE RECEPCIÓN: 18/04/2019 FECHA DE APROBACIÓN: 04/12/2019 FECHA PUBLICACIÓN: 27/02/2020

1. Hospital Vicente Corral Moscoso

\begin{tabular}{l|l} 
Caso & Clinical \\
Clínico & Case
\end{tabular}

DOl: https://doi.org/10.18537/RFCM.37.03.07

Correspondencia:

eripao123ahotmail.com

Dirección:

Calle Montalvo. Misicata

Código Postal:

010151

Celular:

0988309295

Cuenca - Ecuador

\section{RESUMEN}

La enfermedad de Wilson es una condición genética autosómica recesiva poco frecuente. Se ha identificado el gen ATP7B como el que codifica la proteína transportadora de cobre y su deficiencia lleva al acúmulo del metal en el cerebro, hígado y otros órganos vitales. Su diagnóstico clínico precoz es esencial para mejorar la calidad de vida del paciente. A continuación, se presenta el caso de un paciente de 20 años, masculino, con un cuadro clínico de 2 años de evolución de desinhibición, impulsividad, anartria y apraxia de la marcha, movimientos distónicos faciales y en 4 extremidades. Al examen físico se evidenció el anillo de Kayser Flescher a nivel ocular. En Resonancia Magnética Encefálica hiperintensidad en ganglios de la base y mesencéfalo en T2. Ceruloplasmina en suero $4.08 \mathrm{mg} / \mathrm{dL}$. Cobre sérico $26.03 \mathrm{ug} / \mathrm{dL}$ y cobre en orina de 24 horas $224.30 \mathrm{ug} / 24 \mathrm{~h}$. Se confirma el diagnóstico de Enfermedad de Wilson, tratándose con D- Penicilamina, evidenciándose una evolución adecuada, con mejoría notable del cuadro neurológico. El tratamiento precoz permite una evolución favorable temprana del paciente, disminuyendo las secuelas neurológicas secundarias a la enfermedad; de ahí la importancia del reporte del presente caso.

Palabras Clave: informes de casos, degeneración hepatolenticular; atpasas transportadoras de cobre; ceruloplasmina. 


\section{ABSTRACT}

\section{Background}

Wilson's disease is a rare autosomal recessive genetic condition. The ATP7B gene has been identified as the one that encodes the copper transport protein and its deficiency leads to the accumulation of metal in the brain, liver and other vital organs. Your early clinical diagnosis is essential to improve the quality of life of the patient. Following we present the clinical case of a 20 -year-old male patient who since 2 years ago, presented disinhibition, impulsivity, anartria and gait apraxia, facial dystonic movements and in extremities. To the physical exam, Kayser Flescher ring was present. In Brain Magnetic Resonance hyperintensity in Basal Ganglia and Midbrain. Serum Ceruloplasmin 4.08. Serum Copper 26.03. Urinary Cupper 224.30. The diagnosis of Wilson's disease is confirmed, treating with D-Penicillamine, evidencing an adequate evolution, with notable improvement of the neurological symptoms. Early treatment allows a favorable early evolution of the patient, reducing the neurological sequelae secondary to the disease; so that the importance of the report of this case.

Key words: case reports, hepatolenticular degeneration; copper-transporting atpases; ceruloplasmin.

\section{INTRODUCCIÓN}

La enfermedad de Wilson es un trastorno hereditario autosómico recesivo del metabolismo del cobre no curable pero potencialmente tratable, se caracteriza por la acumulación patológica del cobre. Es causada por la mutación en el gen ATP7B, que codifica una ATPasa transportadora de cobre transmembrana, lo que lleva a una alteración de la homeostasis del cobre y la sobrecarga del mismo en el hígado, el cerebro y otros órganos [1].

La enfermedad de Wilson se encuentra en todo el mundo, con una prevalencia estimada de 0.5 - 1 caso por cada 30,000 nacidos vivos en la mayoría de las poblaciones. En la isla de Creta (ciudad de Heraklion), se registró una prevalencia de 1 por cada 15 nacimientos. El aumento en la prevalencia fue probablemente debido a las altas tasas de consanguinidad en el área aislada [2].

Algunos estudios sugieren que los hombres y las mujeres están igualmente afectados, aunque las mujeres son más propensas que los hombres a desarrollar insuficiencia hepática aguda debido a la enfermedad de Wilson [3]. Sin embargo, un gran estudio de registro de 627 pacientes con enfermedad de Wilson encontró que había un ligero predominio masculino (52\%) [4]. Al momento del diagnóstico, entre los pacientes que presentaban síntomas, los hombres tenían más probabilidades que las mujeres de tener una enfermedad neuropsiquiátrica y eran menos propensos a tener enfermedad hepática [5].

Las manifestaciones clínicas son muy diversas (Tabla № 1). Generalmente, en la primera década de vida predominan las manifestaciones hepáticas; no obstante, después de los 20 años, el $75 \%$ de los pacientes presentan síntomas neurológicos solamente, y el otro $25 \%$ denotan tanto manifestaciones hepáticas como neuropsiquiátricas [6]. 


\section{Tabla No 1}

Principales manifestaciones clínicas de la enfermedad de Wilson

\begin{tabular}{|c|c|}
\hline \multirow{6}{*}{ Hepáticas } & Hepatomegalia asintomática \\
\hline & Elevada actividad en suero de las aminotransferasas (AST, ALT) \\
\hline & Hígado graso \\
\hline & Hepatitis aguda \\
\hline & Cirrosis (compensada o descompensada) \\
\hline & Hepatitis fulminante \\
\hline \multirow{4}{*}{ Neurológicas } & Movimientos involuntarios (temblores, pérdida de coordinación motora) \\
\hline & Migrañas, cefaleas, insomnio, pérdida de la memoria \\
\hline & Microfagia, problemas en el lenguaje \\
\hline & Demencia \\
\hline \multirow{3}{*}{ Psiquiátricas } & Depresión \\
\hline & Cambios de personalidad \\
\hline & Psicosis, ansiedad \\
\hline \multirow{6}{*}{ Otros sistemas } & Problemas renales: aminoaciduria, nefrolitiasis \\
\hline & Osteoporosis prematura, artritis \\
\hline & Miocardiopatías \\
\hline & Hipoparatiroidismo \\
\hline & Pancreatitis \\
\hline & Irregularidad menstrual, infertilidad \\
\hline
\end{tabular}

Elaborado por: los autores

Fuente: Feoktistova Feoktistova YC. Enfermedad de Wilson. Rev Med Electrón [internet]. 2016; 38 (1): 57 - 66.

El diagnóstico se establece a partir de los antecedentes familiares, síntomas y signos clínicos (hepáticos, neurológicos, psiquiátricos, oftalmológicos), dosificación del metabolismo del cobre y análisis de ADN de ATP7B.
Para el diagnóstico de la enfermedad se ha propuesto el sistema de puntuación expuesto en la Tabla $N^{\circ} 2$ [7], siendo de especial utilidad la dosificación de cobre y ceruloplasmina sérica (Tabla $\mathrm{N}^{\circ} 3$ ). 


\section{Tabla No 2}

Sistema de puntuación de Leipzig para el diagnóstico de la enfermedad de Wilson

\begin{tabular}{|c|c|c|c|}
\hline Síntomas y signos & & Otras pruebas & \\
\hline Anillo de Kayser - Fleischer & & Cobre intrahepático & \\
\hline Presente & 2 & $>250$ ug/g peso seco & 2 \\
\hline \multirow[t]{2}{*}{ Ausente } & 0 & $50-250 \mathrm{ug} / \mathrm{g}$ peso seco & 1 \\
\hline & & Normal (< 50 ug/g peso seco) & -1 \\
\hline Síntomas neurológicos & & Orceína + & 1 \\
\hline Graves & 2 & & \\
\hline Leves & 1 & Cobre urinario & \\
\hline \multirow[t]{2}{*}{ Ausencia } & 0 & $<100 \mathrm{ug} / 24 \mathrm{~h}$ & 0 \\
\hline & & $100-200 u g / 24 h$ & 1 \\
\hline Ceruloplasmina sérica & & $>200 \mathrm{ug} / 24 \mathrm{~h}$ & 2 \\
\hline Normal (> 20 mg/dl) & 0 & $>1000$ ug / 24 h post $D$ - penicilamina & 2 \\
\hline $10-20 \mathrm{mg} / \mathrm{dl}$ & 1 & & \\
\hline \multirow[t]{2}{*}{$<10 \mathrm{mg} / \mathrm{dl}$} & 2 & Análisis genético & \\
\hline & & Mutación de 2 alelos & 4 \\
\hline Anemia hemolítica Coombs negativ & & Mutación de un alelo & 1 \\
\hline Presente & 1 & Ninguna mutación & 0 \\
\hline Ausente & 0 & & \\
\hline
\end{tabular}

\begin{tabular}{ll}
\hline Puntuación total & Valoración \\
\hline 4 & Diagnóstico de enfermedad de Wilson \\
\hline 3 & Diagnóstico posible. Se necesitan más pruebas \\
\hline 2 & $\begin{array}{l}\text { Diagnóstico de enfermedad de Wilson muy } \\
\text { improbable. }\end{array}$ \\
\hline
\end{tabular}

Elaborado por: Los autores

Fuente: Ochoa A, Ibáñez L, Rodríguez M, Pajares J, Clemente G. Enfermedad de Wilson: espectro clínico de la enfermedad hepática. Rev Gastroenterol Hepatol. 2013;36(2):86-91 [7]. 


\section{Tabla $N^{0} 3$}

Parámetros bioquímicos en adultos normales y con enfermedad de Wilson

\begin{tabular}{|c|c|c|}
\hline Prueba & Enfermedad de Wilson & $\begin{array}{l}\text { Límites normales en } \\
\text { adultos }\end{array}$ \\
\hline $\begin{array}{l}\text { Ceruplasmina } \\
\text { Sérica }\end{array}$ & $<20 \mathrm{mg} / \mathrm{dL}$ & $18-35 \mathrm{mg} / \mathrm{dL}$ \\
\hline Cobre sérico & $3-10 \mathrm{umol} / \mathrm{L}$ & $11-22$ umol/L \\
\hline Cobre urinario & $>40$ ug/día & $20-40$ ug / dìa \\
\hline Cobre hepático & $\begin{array}{c}>200 \mathrm{ug} / \mathrm{g} \text { de peso } \\
\text { seco }\end{array}$ & $20-50 \mathrm{ug} / \mathrm{g}$ de peso seco \\
\hline
\end{tabular}

Elaborado por: Los autores

Fuente: Herrera E, Paola Y, Ruiz M, Manuel L, Gutiérrez R, Carlos J. Wilson's disease: a review. Rev latreia. 2010;23(1):58-66 [8]

Entre los pilares del tratamiento se incluyen la dieta, terapia de quelación y sales de zinc, que revierten la sobrecarga de cobre por diferentes mecanismos. Adicionalmente, el trasplante de hígado está indicado en casos seleccionados, siendo controversial en el caso de pacientes con síntomas neurológicos. Los nuevos agentes, como las sales de tetratiomolibdato, se están investigando actualmente en ensayos clínicos, y las terapias genéticas se están probando en modelos animales. Con el diagnóstico y tratamiento tempranos, el pronóstico es bueno; sin embargo, un problema importante es diagnosticar a los pacientes antes de la aparición de síntomas graves $[9,10]$.

\section{CASO CLÍNICO}

Paciente de 20 años, masculino, diestro, soltero, con secundaria incompleta, residente en Cuenca, con antecedente personal de hepatopatía no especificada a los 14 años, antecedentes familiares de 3 hermanas que fallecen a las edades de 13, 14, 16 años respectivamente por enfermedad hepática no especificada, sin ingesta de medicamentos, alcohol o herbolaria. Desde hace 2 años progresivamente presenta dificultad para la marcha y movimientos distales de 4 extremidades, a los 6 meses se suma trastorno del lenguaje con disminución de la fluencia hasta la pérdida completa del mismo, sialorrea y disfagia.

Examen físico: desinhibición, impulsividad, anartria, escleras ligeramente ictéricas, anillo café de $3 \mathrm{~mm}$ en $360^{\circ}$ (Kayser Fleischer, Imagen $N^{\circ} 1$ ), catarata en girasol bilateral, velo del paladar simétrico con reflejos faríngeos conservados. Apraxia de la marcha, movimientos distónicos oromandibulares y distal en extremidades. Llama la atención varias masas blandas, móviles, no dolorosas en extremidades.

\section{Imágen $N^{0} 1$}

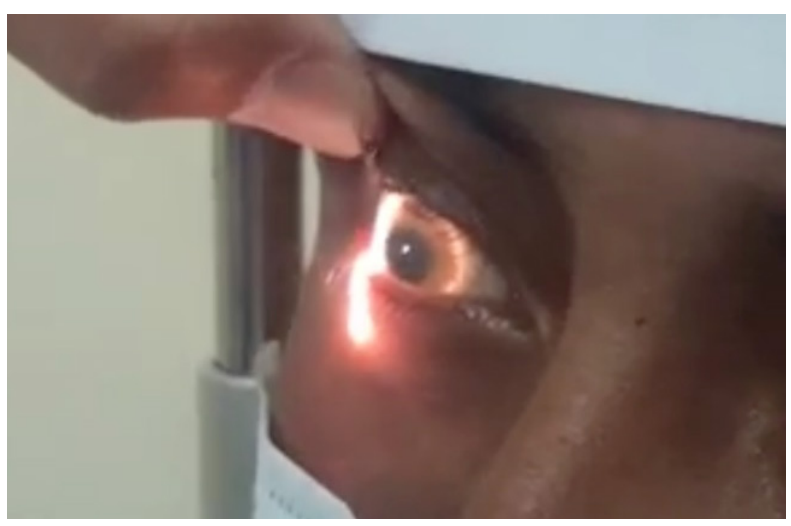

Anillo de Kayser Fleischer, secundario a la acumulación de hierro a nivel de la córnea, característico de la Enfermedad de Wilson.

En los exámenes complementarios se evidenció pancitopenia, tiempos de coagulación prolongados, hiperbilirrubinemia directa, elevación de transaminasas por encima de tres veces el valor normal, con aumento de la fosfatasa alcalina. La ecografía abdominal demostró disminución del tamaño de hígado con líquido libre en espacio de Morrison, es- 
plenomegalia. La biopsia hepática reportó cirrosis hepática con puntuación Child Pugh B, Meld 14.

En la Resonancia Magnética Nuclear encefálica (Imagen $\mathrm{N}^{\circ} 2$ ) se observó un quiste sub- aracnoideo a nivel temporal bilateral; en T2 hiperintensidad a nivel de ganglios de la base y tálamo al igual que en secuencia FLAIR, a nivel de tallo el "signo de la cara de panda" (face of Panda sign).

\section{Imágen $\mathrm{N}^{\circ} 2$}
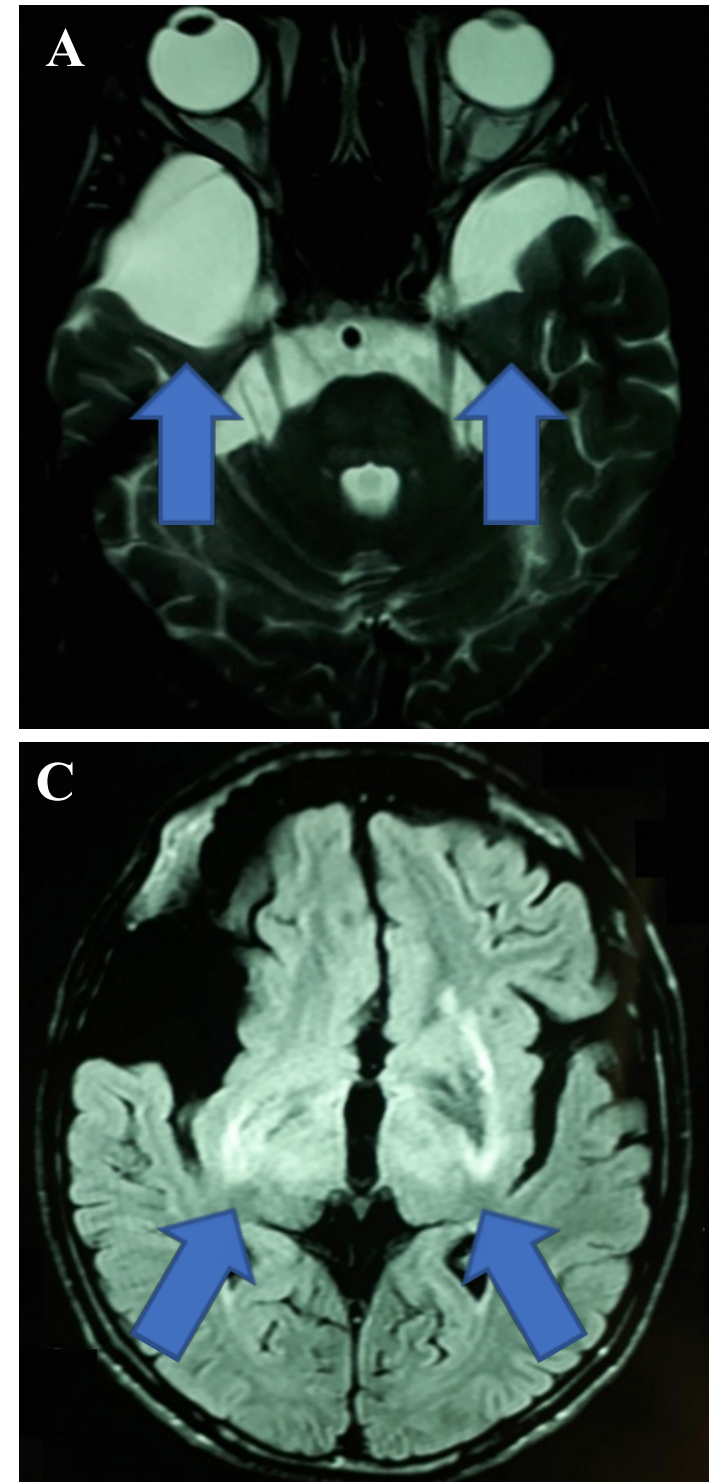

Resonancia Magnética Nuclear encefálica, en la que se observa quiste a nivel temporal bilateral (A), con hiperintensidad en ganglios de la base en T2 y tálamo en secuencia $\operatorname{FLAIR}(B, C)$. Signo de la cara de panda a nivel del mesencéfalo, con visualización de los núcleos rojos y la sustancia negra, rodeadas por áreas hiperintensas en T2 (D).

El resultado de ceruloplasmina sérica fue $4.08 \mathrm{mg} /$ dl (Referencial 22-58 mg/dl), cobre sérico 26.03
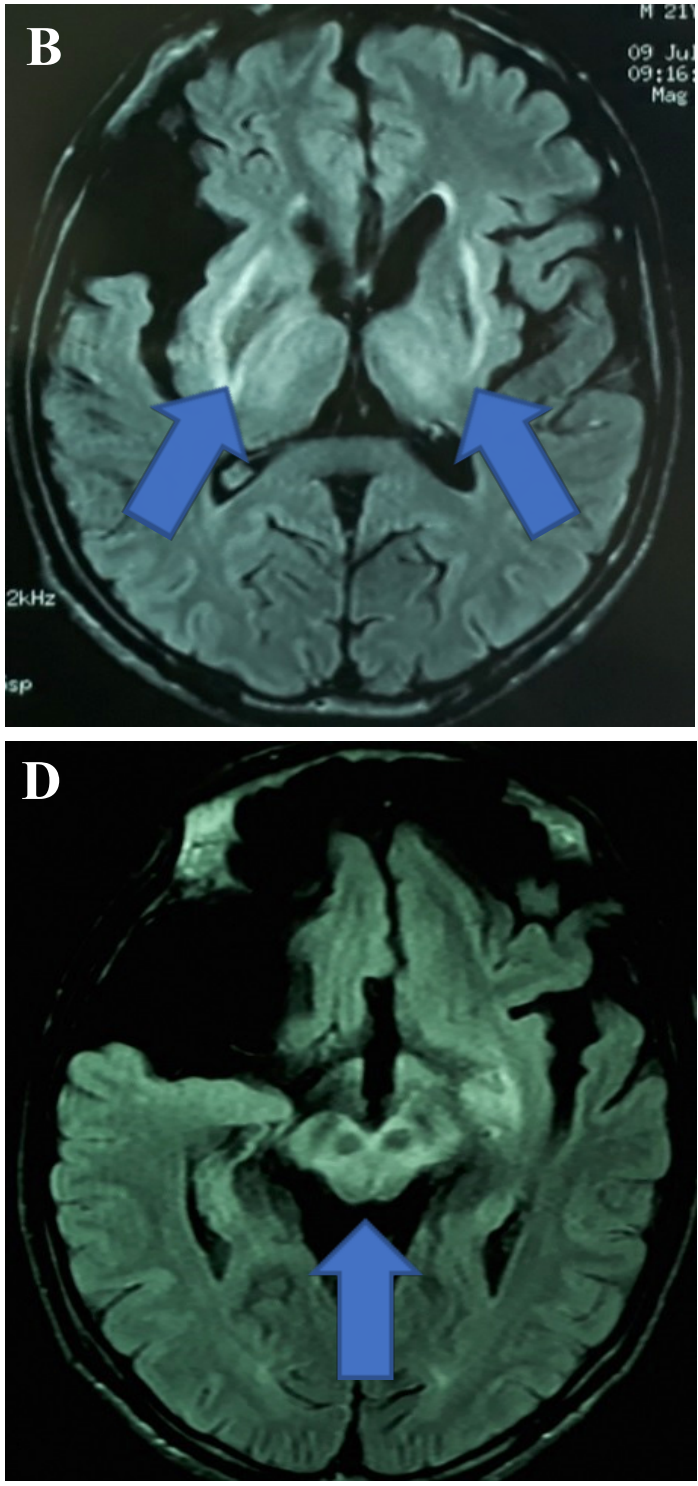

ug/dl (referencial 70-175 ug/dl), cobre en orina de 24 horas 224.30 ug/24h (referencial <60 ug/24h).

\section{EVOLUCIÓN}

El paciente fue valorado por primera ocasión $17 / 08 / 2017$ por la consulta externa con antecedentes familiares de enfermedad hepática y mortalidad por la misma de 3 hermanas, con clínica 
neurológica, psiquiátrica , hepática, oftalmológica (anillo de Kayser Flescher) se plantea como hipótesis diagnóstica Enfermedad de Wilson por lo que se realiza complementarios en los que se encuentra: cirrosis hepática, ceruloplasmina y cobre sérico bajo y cobre en orina elevado; imagen con hiperintensidad en T2 y FLAIR en ganglios de la base y tálamo, además del signo de panda. Con escala de puntuación para enfermedad de Wilson de 9 se inicia tratamiento a base de D- penicilamina. En las primeras 48 horas como efecto adverso presentó fiebre que se controla con antipiréticos. Posteriormente se sube dosis de fármaco presentando como reacción adversa deterioro de cuadro psiquiátrico (psicosis) por lo que se regresa a dosis anterior. Control de Cobre en orina de 24 horas inicial de $224.30 \mathrm{ug} / 24 \mathrm{~h}$ posterior a Terapia farmacológica $463.68 \mathrm{ug} / 24 \mathrm{~h}$

En cuanto a la pancitopenia que probablemente se sospechó sea secundaria a cirrosis hepática, se interconsulta a reumatología con resultados de ANAS, Anti-ds DNA, IgG, complemento C3 y C4 normales, descartando patología autoinmune asociada.

Además se valoró a paciente por cirugía general realizando exéresis de masas con biopsia compatible con lipomas.

Durante su evolución paciente con ascitis a tensión como complicación de enfermedad hepática, que requirió paracentesis evacuatoria y diuréticos.

Paciente con evaluaciones periódicas por los servicios de neurología, genética, gastroenterología, se evidencia progresivamente mejoría de cuadro neurológico: da pasos con apoyo, remisión completa de la disfagia y distonía. Se mantiene con discreto temblor de miembro superior izquierdo.

\section{DISCUSIÓN}

La enfermedad de Wilson es una enfermedad por depósito de cobre poco frecuente, con herencia autosómica recesiva. La clínica es variada con compromiso hepático, neurológico, psiquiátrico, oftalmológico y de otros órganos vitales. Desde la primera consulta del paciente con los antecedentes familiares de enfermedad hepática y trastornos del movimiento, además de síntomas de todos los sistemas antes mencionados, se sospechó enfermedad de Wilson solicitando complementarios que apoyen presunción clínica además de valoración por especialidades comprobando compromiso hepático (cirrosis y ascitis), oftalmológico (anillo de Kayser Flescher).

En cuanto a la prevalencia de la enfermedad de Wilson a nivel de Centro y América del Sur se han reportado casos aislados siendo la prevalencia más elevada en Costa Rica, Cuba y Brasil. A nivel mundial la mayor prevalencia se registra en Alemania, Japón y Creta [10,11].

El espectro clínico es amplio pero generalmente en un $40-50 \%$ de pacientes los síntomas iniciales son hepáticos y tienden a aparecer a una etapa temprana entre los 11 y 14 años [12].

Entre las manifestaciones hepáticas más frecuentes están: cirrosis, hepatitis, y en el contexto de enfermedad hepática la anemia hemolítica podría ser un indicio de la enfermedad. La hepatitis fulminante se presenta solo en un $5 \%$ de casos sobre todo en mujeres jóvenes [13].

Comparativamente con nuestro paciente se tiene el antecedente de enfermedad hepática que fue considerada como viral en el 2014, además llama la atención la muerte de las hermanas con enfermedad hepática fulminante en concordancia con la bibliografía que sugiere que los casos de hepatitis fulminante son más frecuente en el sexo femenino.

En un $40 \%$ de pacientes los síntomas neurológicos se presentan desde el inicio siendo el más frecuente el temblor y la distonía, que cuando es a nivel facial produce la llamada risa sardónica [14]. La distonía fue una de las manifestaciones principales con la característica risa sardónica y el temblor que al momento persiste en miembro superior izquierdo.

En cuanto a las manifestaciones psiquiátricas se pueden presentar en un $30 \%$ de pacientes y varían desde trastornos de aprendizaje hasta psicosis y riesgo de suicidio $[15,16]$.

En nuestro caso se detectó depresión y asociado a fármaco psicosis que cedió con la diminución de quelante.

El anillo de Kayser Flescher por acúmulo de cobre a nivel corneal se presenta en $100 \%$ de los pacientes con manifestaciones neurológicas, $86 \%$ de pacientes con manifestaciones hepáticas y $50 \%$ de pacientes con manifestaciones psiquiátricas. 
Menos frecuente cataratas en girasol [17]. Al inicio de la atención estos síntomas oftalmológicos no fueron encontrados, en Mayo de este año estos signos fueron evidentes.

Los lipomas subcutáneos tienen mayor prevalencia en pacientes con enfermedad de Wilson que en la población general, con ubicación principal en tronco y extremidades superiores, ni la presentación ni el tratamiento influyeron en la presencia o curso de los mismos y se considera como un buen parámetro para diagnóstico diferencial [18]. En un estudio a nivel terciario el $80 \%$ de los pacientes presentaron al menos un lipoma subcutáneo.

En el caso del paciente encontramos múltiples masas blandas no dolorosas en extremidades y tronco que con biopsia se llegó a la conclusión que se trataban de lipomas en correlación a bibliografía.

Debe pensarse en el diagnóstico de la enfermedad de Wilson en personas con enfermedad hepática de causa no conocida especialmente jóvenes con trastornos del movimiento. La prueba específica es la genética que comprueba la mutación del gen ATP7B. En la práctica clínica el diagnóstico se basa en la combinación de manifestaciones clínicas y pruebas de laboratorio (ceruloplasmina baja y cupriuria elevada) ninguna de ellas individualmente permite confirmar o excluir el diagnóstico. En una reunión de expertos en Leipzig en el 2001 se propuso un sistema de puntuación que ha facilitado notablemente el diagnóstico. Este sistema ha sido validado en la población adulta [19].

En el paciente el diagnóstico se basó en la clínica y las pruebas complementarias. Con una puntuación de 9 .

Para el tratamiento de la enfermedad de Wilson tenemos varios pilares entre los que encontramos dieta baja en cobre, quelantes del cobre que promueven la excreción renal del cobre y un bloqueador de la absorción intestinal que además estimula la producción citoplasmática de metalotioneína que fija el cobre en el citoplasma de los hepatocitos de forma no tóxica y actúa como quelante endógeno como es el zinc [20].

El tratamiento debe mantenerse toda la vida, y en los casos de falla hepática severa se recomienda el trasplante hepático incluso en presencia de enfermedad neurológica y psiquiátrica es decir presentación mixta en las que el trasplante hepático ha contribuido a la mejoría de las mismas [21,22].

En el caso del paciente como quelante se utilizó D penicilamina con mejoría neurológica importante y precoz. En el país se conocen 7 casos de pacientes con esta patología: 1 paciente fallece por hemorragia durante biopsia hepática, 3 pacientes sexo femenino fallecen con enfermedad hepática son hermanas de nuestro paciente, 2 pacientes en tratamiento en Quito, 1 paciente con trasplante hepático con buena respuesta al tratamiento. Los datos anteriores no están publicados.

En el país al momento no hay documentación estadística de esta patología quizá por ser infradiagnosticada. La relevancia de la presentación de este caso es fundamental tanto para registro de enfermedad y posteriores estudios como para incentivar ante la sintomatología descrita considerar como sospecha esta patología.

Cabe mencionar que ante la sospecha clínica de esta patología es importante el estudio de la familia para prevenir y tratar tempranamente a fin de disminuir la discapacidad y manejo multidisciplinario por las complicaciones que se presentan por la enfermedad y por el tratamiento.

\section{CONCLUSIONES}

La enfermedad de Wilson tiene baja incidencia a nivel mundial pero debe ser considerada en un paciente adulto joven con enfermedad hepática, neurológica y psiquiátrica.

La posibilidad de infradiagnostico puede ser elevada tanto por la variabilidad de la presentación clínica como por el antecedente de consanguinidad en nuestro país condición que favorece a la presentación de este tipo de enfermedades.

El diagnóstico temprano y manejo multidisciplinario son la clave junto con el seguimiento periódico a fin de evitar complicaciones y brindar a los pacientes una mejor calidad de vida.

Es importante el estudio a la familia y el consejo genético. 
Los pacientes con enfermedad hepática e incluso con presentación mixta de la enfermedad se benefician del trasplante hepático.

El factor de predicción más útil para hacer un diagnóstico precoz y un tratamiento eficaz es "sospechar la enfermedad".

\section{ASPECTOS BIOÉTICOS}

El presente trabajo se realizó bajo el consentimiento informado del paciente, con total confidencialidad de datos personales.

\section{INFORMACIÓN DE LOS AUTORES}

- Guartazaca Guerrero Erika Paola. Especialista en Primer Grado en Neurología. Hospital Vicente Corral Moscoso. Clínica. Neurología. Cuenca. Azuay. Ecuador.

e-mail: eripao123@hotmail.com

ORCID: https://orcid.org/0000-0002-6510-1107

- León Domínguez Cristina Elizabeth. Especialista Primer Grado en Genética Clínica. Hospital Vicente Corral Moscoso. Pediatría. Cuenca. Azuay. Ecuador.

e-mail: crieledo@gmail.com

ORCID: https://orcid.org/0000-0003-4055-3868

- Orellana Cobos Danilo Fernando. Médico. Posgradista en Medicina Interna. Hospital Vicente Corral Moscoso. Clínica. Medicina Interna. Cuenca. Azuay. Ecuador.

e-mail: dforellanac891@hotmail.com

ORCID: http://orcid.org/0000-0001-6269-5512

\section{CONTRIBUCIÓN DE LOS AUTORES}

EG, CL, DO: Realizaron el manejo clínico del paciente, la recolección de datos, revisión bibliográfica y redacción del manuscrito. EG, CL: realizaron el análisis crítico del artículo. Todos los autores leyeron y aprobaron la versión final del manuscrito.

\section{CONFLICTO DE INTERESES}

Los autores no reportan conflictos de intereses.

\section{FUENTES DE FINANCIAMIENTO}

Autofinanciado.

\section{REFERENCIAS BIBLIOGRÁFICAS}

1. Członkowska A, Litwin T, Dusek P, Ferenci P, Lutsenko S, Medici V, et al. Wilson disease. Nat Rev Dis Primer. 2018;4(1):21.

2. Parkash O, Ayub A, Jafri W, Alishah SH, Hamid S. Wilson's disease: Experience at a tertiary care hospital. J Coll Physicians Surg-Pak JCPSP. 2013;23(7):525-6.

3. Assis F, Araujo D, Oliveira I, Cardoso O. Small fiber dysfunction in patients with Wilson's disease. Rev Arq Neuro Psiquiatr. 2014; 72(8): 592 - 95.

4. Schilsky M. Wilson disease: Epidemiology and pathogenesis. UpToDate [Internet]. 2018. Disponible en: https://www.uptodate. com/contents/wilson-disease-epidemiologyand-pathogenesis/abstract/2

5. Altraif I, Handoo FA, Al Ghamdi H, Aljumah $A$, Al Jumah M, Afzal M. Presentation, diagnosis and outcome of predominantly hepatic Wilson's disease in adult Saudi patients: a single centre experience. Saudi J Gastroenterol Off J Saudi Gastroenterol Assoc. 2012;18(5):334-8.

6. Guerra L, Ortega F, Sumire J, Cok J. Enfermedad de Wilson: forma hepática. Rev Gastroenterol Perú. 2015;35(4):361-5.

7. Ochoa $A$, Ibáñez $L$, Rodriguez $C$, Vega $M$, Pajares J, Clemente Ricote G. Enfermedad de Wilson: espectro clínico de la enfermedad hepática. Gastroenterol Hepatol. 2013;36(2):86-91.

8. Espinoza Y, Muñoz L, Restrepo JC. Enfermedad de Wilson: revisión del tema. latreia. 2010;23(1):58-66

9. Feoktistova Y. Enfermedad de Wilson. Rev Med Electrón [internet]. 2016; 38(1): 57 - 66. Disponible en: http://scielo.sld.cu/pdf/rme/ v38n1/rme060116.pdf

10. Liu J, Luan J, Zhou X, Cui Y, Han J. Epidemiology, diagnosis, and treatment of Wilson's disease. Intractable Rare Dis Res. 2017;6(4):249-55. 
11. Hevia F, Alvarado I, Sanabria A, Sánchez M, Meza L, Parajeles A, et al. National alliance for Wilson's disease: health policy in Costa Rica. Hepatol Med Policy. 2016;2(1):5.

12. Pfeiffer RF. Wilson Disease. Lifelong Learning in Neurology [internet]. 2016;22:1246-61. Disponible en: https://www.ncbi.nlm.nih.gov/ pubmed/27495207

13. Bandmann O, Weiss KH, Kaler SG. Wilson's disease and other neurological copper disorders. Lancet Neurol. 2015;14(1):103-13.

14. Machado A, Chien HF, Deguti MM, Cançado E, Azevedo RS, Scaff M, et al. Neurological manifestations in Wilson's disease: Report of 119 cases. Mov Disord Off J Mov Disord Soc. 2006;21(12):2192-6.

15. Rodrigues ACT, Dalgalarrondo P. Neuropsychiatric disturbances in Wilson's disease and use of electroconvulsive therapy: case report. Rev Arq Neuropsiquiatr. 2003;61(3B):876-80.

16. Zimbrean PC, Schilsky ML. The spectrum of psychiatric symptoms in Wilson's disease: treatment and prognostic considerations. Am J Psychiatry. 2015;172(11):1068-72.

17. Ram J, Gupta A. Kayser-Fleischer ring and sunflower cataract in Wilson disease. JAMA Ophthalmol. 2014;132(7):873.

18. Schaefer M, Gotthardt DN, Didion C, Stremmel W, Weiss $\mathrm{KH}$. Increased Prevalence of Subcutaneous Lipomas in Patients With Wilson Disease. J Clin Gastroenterol. 2015;49(7):61-63.

19. Bruguera $\mathrm{M}$, Abraldes JG. Common problems in the diagnosis and treatment of Wilson's disease. Gastroenterol Hepatol. 2013;36(5):316-25.

20. Gromadzka G, Grażyna G, KarpińskaA, Agata K, Przybyłkowski A, Adam P, et al. Treatment with D-penicillamine or zinc sulphate affects copper metabolism and improves but not normalizes antioxidant capacity parameters in Wilson disease. Biometals Int J Role Met Ions Biol Biochem Med. 2014;27(1):207-15.
21. Yagci MA, Tardu A, Karagul S, Ertugrul I, Ince V, Kirmizi S, et al. Influence of Liver Transplantation on Neuropsychiatric Manifestations of Wilson Disease. Transplant Proc. 2015;47(5):1469-73.

22. Członkowska A, Litwin T, Karliński M, Dziezyc K, Chabik G, Czerska M. D-penicillamine versus zinc sulfate as first-line therapy for Wilson's disease. Eur J Neurol. 2014;21(4):599-606. 